\title{
On extensions of Pascal's theorem (Second paper) Paul Serret's theorem
}

\author{
By H. W. Richmond.
}

(Received 24th November, 1937. Read 3rd December, 1937.)

1. The circumstances explained in the footnote on p. 61 of the former paper with this title ${ }^{1}$ might well have necessitated a re-writing of the whole. Fortunately it appears that only a few short comments are required. For example it may be noted that the first question in $\$ 4$ can be answered by counting the degrees of freedom in the two configurations. Eight points of a twisted cubic have freedom 20; four pairs of planes drawn at random through four lines of a regulus have freedom 21; therefore the eight planes of the second paragraph of $\S 4$ cannot always lead back to the eight points of $\S 2$. This is corroborated by the corresponding numbers in [4], which are 31 and 34 .

The extensions of Pascal's (or Brianchon's) theorem connect two configurations in $[n]$ to each of which the term "associated" has been applied; sets of $2 n+2$ associated points, and sets of $n+1$ associated lines; more precisely, each of them is connected with the dual of the other. We shall now consider the two configurations. separately.

On sets of $2 n+2$ associated points in $[n]$.

2. A set of $2 n+2$ points in $[n]$ is said to be an associated set ${ }^{2}$ when every quadric passing through all but one of the points must pass through the last point. Such sets have been studied by several writers; but none of them recognise that the fundamental property of the sets is a very special case of an important general theorem ${ }^{3}$.

1 Proceedings of the Edinburgh Mathematical Society (2), 5, pp. 55-62.

'See Encyk. d. math. Wiss. III 2 2A, pp. 836-837.

${ }^{3}$ It was published in 1869 in a book bearing the misleading title Géométrie de Direction, which consisted almost entirely of deductions from the theorem. Serret also used the reciprocal theorem on sets of lines, and of its extension to three dimensions ; but at that date naturally did not mention the equally obvious extension to space of many dimensions. The mode of its publication explains and partly excuses the neglect of the theorem, but it is noteworthy that W. K. Clifford recognised its importance. (Collected Papers, XIV and XV). 
due to M. Paul Serret. Indeed the theorem has been so persistently overlooked that it is worth while to enunciate it and consider it.

Paul Serret's Theorem. In order that a set of points may be so related that every curve of order $s$ which passes through all but one of the points must pass through the remaining point, it is necessary and sufficient that the $s^{\text {th }}$ powers of their distances from an arbitrary line should satisfy a linear homogeneous relation

$$
k_{1} P_{1}^{s}+k_{2} P_{2}^{s}+k_{3} P_{3}^{s}+\ldots \equiv 0 \ldots(\Sigma)
$$

This statement of the theorem is Clifford's. Clearly it would be better to describe $P_{1}=0, P_{2}=0$, etc. as the equations (in tangential coordinates) of the points of the set. It is unnecessary to state the reciprocal theorem. For the analogous theorems in space of three or more dimensions, read surface or primal in place of curve, and plane or prime in place of line. (The special case when $s$ is 2 had been given by Serret in 1865 and by Hesse in 1866). Serret does not even state his theorem in general terms, and Clifford accepts it without formal proof: actually a lemma (easily proved by induction) is all that is needed.

When nothing is known concerning a number of quantities except that they satisfy a number of given homogeneous linear relations, the most general linear relation necessarily satisfied by the quantities is a sum of multiples of (some or all of) the given relations.

The condition that a curve of order $s$ should pass through a point whose coordinates are known is a homogeneous linear relation in the unknown coefficients of the equation of the curve; from this the theorem is deduced without difficulty. The lemma is valid even if some of the linear relations are superfluous, being derivable from others. In the application of the lemma to Serret's theorem, if (for this or another reason) one of the set of points does not appear in the formulae, that point does not really belong to the set and must be discarded. In Serret's definition of a set of associated points it is tacitly assumed that none of the constants $k$ vanish. All this can be extended to $[n]$.

For our present purpose $s$ is 2, and the number of points is $2 n+2$ in $n$-dimensional space; the fundamental condition, both necessary and sufficient, that points defined by equations $P_{1}=0$, $P_{2}=0, P_{3}=0, \ldots$ should form an associated set in connection with quadric primals is that values of the constants $k$ should exist which make

$$
k_{1} P_{1}^{2}+k_{2} P_{2}^{2}+k_{3} P_{3}^{2}+\ldots \equiv \equiv
$$


From this the property which all the writers have noticed, viz. that the points are vertices of two self-polar simplexes of a certain quadric, follows at once.

\section{On sets of $n+1$ associated lines in [n].}

3. It is convenient here to adopt the word "sub-prime" to denote a flat space of $n-2$ dimensions in [n], i.e. the space common to two primes, or the dual of a straight line. In order that a line and a sub-prime may intersect, i.e. have one common point and lie in a prime, one condition must be satisfied. A set of $n+1$ lines which form an associated set has the property that every sub-prime which intersects $n$ of them also intersects the remaining line; but this is not a sufficient definition. A line in $[n]$ is defined by $n(n+1) / 2$ coordinates; for lines of an associated set every linear relation satisfied by the coordinates of $n$ of them must be satisfied by the coordinates of the remaining line; every linear complex which includes $n$ of the lines must include the last line. Or, to state the same fact in a more convenient form, it is possible to arrange that the $n+1$ values of each of the $n(n+1) / 2$ coordinates shall have the sum zero. This is a necessary and sufficient condition; if true in one coordinate-system it will remain true however the coordinate-system is changed. This fact leads to a distinctive geometrical property of a set of $n+1$ associated lines.

For, supposing that we have such a set of lines, take points chosen at random, one on each line, as vertices of the simplex of reference, the coordinates being $(X)$ or $(Y)$ for points, $(U)$ or $(V)$ for primes, $P_{r, s}=X_{r} Y_{s}-X_{s} Y_{r}$ for the line joining points $(X),(Y)$. A second point taken anywhere on each line defines the line. Suppose then that the lines join the points

$$
\begin{aligned}
& (1,0,0,0, \ldots) \text { to }\left(c_{11}, c_{12}, c_{13}, c_{14}, \ldots\right) \\
& (0,1,0,0, \ldots) \text { to }\left(c_{21}, c_{22}, c_{23}, c_{24}, \ldots\right) \\
& (0,0,1,0, \ldots) \text { to }\left(c_{31}, c_{32}, c_{33}, c_{34}, \ldots\right) \text { etc., etc. }
\end{aligned}
$$

The line-coordinates $P_{r, 8}$ may now be found, and it is seen that each coordinate vanishes for all but two of the lines: $P_{1,2}$ is $c_{12}$ for the first line, is $-c_{21}$, for the second line, and is zero for all the rest. Now since we are concerned only with the ratios of the coordinates of points, it is permissible to introduce a factor into the $c$ 's of the second, third, and later points so as to make $c_{21}=c_{12}, c_{31}=c_{13}$, $c_{41}=c_{14} \ldots$ When this is done the sum of the values of the coordinates $P_{12}$ for all the lines vanishes; and so do those of $P_{13}, P_{14}$, 
$P_{15}$, etc. But for coordinates (such as $P_{23}$ ) in which the suffix 1 does not enter, the sum $\left(c_{23}-c_{32}\right)$ will not vanish unless $c_{32}=c_{23}$. For the lines to form an associated set the symmetry we imposed upon the first line and column must extend over the whole square array of coordinates $c: c_{s r}$ must equal $c_{r s}$ throughout.

This being so, it is clear that the quadric whose tangential equation is

$$
\Sigma c_{r r} U_{r}^{2}+2 \Sigma c_{r s} U_{r} U_{s}=0
$$

has the point $\left(c_{11}, c_{12}, c_{13}, c_{14}, \ldots\right)$ as pole of the prime $X_{1}=0$, and so for the other points and primes. We have now a geometrical condition. Given a set of $n+1$ lines in $[n]$; take at random a point on each line as vertices of a simplex; take a second point at random on each line to form a second simplex. In order that the lines should form an associated set it is necessary and sufficient that a quadric should exist with respect to which the simplexes are conjugate.

\section{On extensions of Pascal's (or Brianchon's) theorem and their converses.}

4. There is an advantage in stating the extension of Brianchon's rather than Pascal's version of the theorem, inasmuch as points, lines and primes alone are involved, and not the unfamiliar subprimes.

"Given in [n] a set of $2 n+2$ associated primes, arrange them in a cycle. Each $n$ consecutive primes in the cycle intersect in a point, giving $2 n+2$ points, vertices of a skew polygon. The $n+1$ lines which join the pairs of opposite vertices of the polygon form a set of associated lines."

"Conversely, given in [n] a set of $n+1$ associated lines, take at random two points on each line and arrange them in a cycle in such a way that two points on the same line are opposite to one another in the cycle. The primes which contain $n$ consecutive points of the cycle form a set of $2 n+2$ associated primes in $[n] . "$

The proofs given in my earlier paper for analogues of Pascal's theorem in [3] and [4] can be extended without difficulty to [n]; by the principle of duality they can be applied to the first of the theorems above. The result obtained in $\S 3$ of the present paper enables us to retrace each step and so establishes the second.

King's College, Cambridge. 